\title{
Reduced miR-125a-5p expression is associated with gastric carcinogenesis through the targeting of E2F3
}

\author{
YANJUN XU ${ }^{1}$, ZHENXIA HUANG $^{2}$ and YUELI LIU ${ }^{2}$ \\ ${ }^{1}$ Department of Chemotherapy, Zhejiang Cancer Hospital, Hangzhou, Zhejiang 310022; \\ ${ }^{2}$ Department of Cell Biology and Program in Molecular Cell Biology, Zhejiang University School of Medicine, \\ Hangzhou, Zhejiang 310058, P.R. China
}

Received September 20, 2013; Accepted April 1, 2014

DOI: $10.3892 / \mathrm{mmr} .2014 .2567$

\begin{abstract}
Emerging evidence suggests that altered expression of microRNAs (miRNAs) is involved in cancer progression. However, the role of $\mathrm{miR}-125 \mathrm{a}-5 \mathrm{p}$ in gastric carcinogenesis remains unknown. Quantitative real-time PCR analysis revealed that the expression of miR-125a-5p was significantly decreased in $>80 \%$ of gastric cancer tissues compared with their adjacent non-tumor tissues, and was markedly reduced in $\sim 95 \%$ of intestinal-type gastric cancer tissues. The downregulated miR-125a-5p was significantly associated with gastric cancer metastasis. Ectopic expression of miR-125a-5p substantially inhibited the proliferation, migration and invasion activities of gastric cancer cells. Furthermore, forced expression of miR-125a-5p repressed the activity of a luciferase reporter carrying the 3'-untranslated (3'-UTR) region of $\mathrm{E} 2 \mathrm{~F} 3$, which was eliminated by mutation of the predicted miR-125a-binding site, indicating that E2F3 may be a potential target gene of miR-125a-5p. These data suggest that by targeting E2F3, miR-125a-5p may be important as a potential tumor suppressor gene in gastric carcinogenesis.
\end{abstract}

\section{Introduction}

MicroRNAs (miRNAs) are a class of single-stranded non-coding RNAs that regulate target gene expression, predominantly by base pairing to the 3'-untranslated (3'-UTR) region of their target mRNAs (1). miRNAs have been linked to carcinogenesis due to their apparent proximity to chromosomal breakpoints and aberrant expression levels in various malignancies. Furthermore, approximately half of miRNA genes are located in cancer-correlated genomic regions or fragile sites (2). A number of studies have provided

Correspondence to: Dr Yanjun Xu, Department of Chemotherapy, Zhejiang Cancer Hospital, Banshan Bridge Guangji Road 38, Hangzhou, Zhejiang 310022, P.R. China

E-mail: 21972483@qq.com

Key words: miR-125a-5p, E2F3, gastric cancer, proliferation, migration, invasion evidence that miRNAs are associated with cancer development and progression, including in gastric (3-5), lung (6), breast $(7,8)$ and hepatocellular carcinoma (9). miRNAs that are upregulated or downregulated in various cancer types are respectively referred to as oncogenic or tumor-suppressor miRNAs (10-12).

MiRNAs have been identified to be associated with the carcinogenesis of human gastric cancer, which ranks as the fourth most common cancer and the second leading cause of cancer-associated mortality worldwide $(13,14)$. miRNAs including miR-150, miR-27a, miR-21, miR-106b and miR-25 are upregulated in gastric cancer, and as oncogenes, they promote proliferation and migration of gastric cancer cells (15-18). Other miRNAs are downregulated in gastric cancer, including miR-218, miR-9, miR-143 and miR-145, and function as possible tumor-suppressor genes to inhibit cell proliferation, migration and metastasis (19-21). However, a number of studies have identified no further evidence for their causative role in gastric carcinogenesis. The mechanisms and functions of the majority of these miRNAs remains to be elucidated.

Recently, we performed miRNA microarray profiling in primary gastric cancer tissues and their adjacent non-tumor tissues and identified a number of miRNAs that were dysregulated in gastric cancer (22). The expression pattern of 6 miRNAs (miR-663, miR-21, miR-25, miR-106a, miR106b and miR-375) in gastric cancer was consistent with other independent studies (18,23-25). For example, we identified that downregulated miR-375 in the majority of gastric cancer tissues was able to suppress gastric cancer cell proliferation and viability, which is confirmed by the data from Moriyama et al (25). In this screening, we also provide evidence that miR-125a-5p, a miRNA with unknown function, appeared to be one of the most downregulated miRNAs in gastric cancer tissues. In the present study, it was confirmed that miR-125a-5p expression was reduced in $>80 \%$ of gastric cancer tissues and we demonstrated that ectopic expression of miR-125a-5p inhibited the proliferation, migration and invasion of gastric cancer cells, suggesting a tumor-suppressive role of miR-125a-5p in gastric carcinogenesis. Furthermore, miR-125a-5p targets E2F3 via its 3'-UTR region, indicating that $\mathrm{E} 2 \mathrm{~F} 3$ may be a potential downstream target of miR-125a-5p in gastric cancer progression. 


\section{Materials and methods}

Tissues and cell lines. The clinical characteristics of all 51 patients in our study are listed in Table I. Gastric tissues were obtained from 51 gastric cancer patients undergoing gastric resection at the Sir Run Run Shaw Hospital (Hangzhou, Zhejiang, China). Written consent was obtained prior to surgery, as described previously (22). The study was approved by the Ethics Comittee of Zhejiang University School of Medicine (Hangzhou, China). The tumor tissues and adjacent non-tumor tissues were quickly separated into two sections following resection. One was immediately frozen in liquid nitrogen for RNA isolation and another was fixed in formalin for pathological examination. Final pathological diagnosis was independently made by at least two professional pathologists.

The source and culture conditions of 6 gastric cancer cell lines (NCI-N87, AGS, MGC-803, HGC-27, SGC-7901 and BGC-823 cells) and one non-malignant gastric epithelial cell line (GES-1 cells) were previously described $(22,26)$.

$R N A$ extraction and quantitative real-time PCR. Total RNA, including low molecular weight RNA from gastric tissue samples and gastric epithelial cells, were isolated using the MirVana $^{\mathrm{TM}}$ miRNA Isolation kit (Ambion, Foster City, CA, USA) according to the manufacturer's instructions. Expression of miR-125a-5p was analyzed using a TaqMan microRNA assay kit (Applied Biosystems, Grand Island, NY, USA). Briefly, for each sample, $10 \mathrm{ng}$ total RNA was reversely transcribed using a TaqMan microRNA reverse transcript kit (PN: 4366597) and a miR-125a-5p specific reverse transcript primer (Applied Biosystems, Foster City, CA, USA). Quantitative real-time PCR was then performed using TaqMan 2X PCR master mix (Applied Biosystems) on an Applied Biosystems 7500 system. All of the miR-125a-5p threshold cycles (Ct values) were normalized to snRNA U6 (RNU6B, Applied Biosystems) and an endogenous control. The normalized values $(\Delta \mathrm{Ct})$ from gastric cancer tissues were subsequently compared with that of their adjacent non-tumor tissues.

Cell proliferation assay. AGS and MGC- 803 cells (4,000 cells/well) were transfected with miR-125a-5p precursor (pre-miR-125a-5p) or a negative control (Applied Biosystems) in 96-well plates using siPORT ${ }^{\mathrm{TM}} \mathrm{NeoF} X^{T M}$ transfection agent (Ambion) following the manufacturer's instructions. At 24, 48 and $72 \mathrm{~h}$ post-transfection, cells were imaged by phase contrast microscopy (Olympus IX81, Olympus Corporation, Tokyo, Japan) and counted manually using a hemocytometer. MTT assays were performed as described previously (26). Each group was conducted in triplicate and all experiments were repeated at least three times independently.

Transwell migration analysis. AGS and MGC-803 cells $(80,000$ cells/well) were transfected with pre-miR-125a-5p or negative control using siPORT NeoFX transfection agent in 24-well plates, following the manufacturer's instructions Following transfection $(24 \mathrm{~h}$ ), the cells were placed in a serumfree medium for another $12 \mathrm{~h}$, chemotactic assays were then conducted in 24-well Transwell inserts with $8 \mu \mathrm{m}$ pore size (Corning Costar Corp.). The cells $(30,000)$ were suspended in $100 \mu \mathrm{l}$ corresponding culture medium without fetal bovine
Table I. Clinical and pathological characteristics of included patient samples and miR-125a-5p expression.

\begin{tabular}{|c|c|c|c|}
\hline \multirow[b]{2}{*}{ Characteristic } & \multicolumn{2}{|c|}{ Expression of miR-125a-5p } & \multirow[b]{2}{*}{ P-value } \\
\hline & $\begin{array}{l}\text { Upregulated } \\
\qquad(n=9)\end{array}$ & $\begin{array}{l}\text { Downregulated } \\
\qquad(n=42)\end{array}$ & \\
\hline \multicolumn{4}{|l|}{ Gender } \\
\hline Male & 8 & 30 & 0.28 \\
\hline Female & 1 & 12 & \\
\hline \multicolumn{4}{|l|}{ Age (years) } \\
\hline$\leq 65$ & 4 & 23 & 0.52 \\
\hline$>65$ & 5 & 19 & \\
\hline \multicolumn{4}{|c|}{ Lauren's classification } \\
\hline Intestinal & 1 & 21 & 0.06 \\
\hline Diffuse & 4 & 7 & \\
\hline Others & 4 & 14 & \\
\hline \multicolumn{4}{|l|}{ Differentiation } \\
\hline Well & 1 & 14 & 0.23 \\
\hline Moderate & 2 & 11 & \\
\hline Low & 6 & 17 & \\
\hline \multicolumn{4}{|l|}{ Invasion depth } \\
\hline $\mathrm{T} 1$ & 0 & 4 & 0.26 \\
\hline $\mathrm{T} 2$ & 2 & 2 & \\
\hline T3 & 7 & 35 & \\
\hline $\mathrm{T} 4$ & 0 & 1 & \\
\hline \multicolumn{4}{|c|}{ Lymph node metastasis } \\
\hline Absent & 3 & 10 & 0.55 \\
\hline Present & 6 & 32 & \\
\hline \multicolumn{4}{|c|}{ Distant metastasis } \\
\hline M0 & 6 & 39 & $0.03^{\mathrm{a}}$ \\
\hline M1 & 3 & 3 & \\
\hline \multicolumn{4}{|l|}{ UICC stage } \\
\hline I & 1 & 5 & 0.82 \\
\hline II & 1 & 6 & \\
\hline III & 4 & 23 & \\
\hline IV & 3 & 8 & \\
\hline
\end{tabular}

${ }^{\mathrm{a}} \mathrm{P}<0.05$. UICC, International Union Against Cancer.

serum (FBS) and loaded into the top chamber of Transwell insert for the migration assay. The bottom chamber was placed with $600 \mu 1$ medium containing 20\% FBS. Migration of cells was allowed to proceed for $12 \mathrm{~h}$ at $37^{\circ} \mathrm{C}$. The cells that migrated into the bottom chamber were then fixed, stained with 4',6-diamidino-2-phenylindole (DAPI) for $2 \mathrm{~min}$, visualized under phase contrast microscope and photographed. Total number of migrated cells in nine randomly selected fields was counted by IPP 6.0 (Image-Pro Plus 6.0) software (Media Cybernetics, Inc. Rockville, MD, USA). All experiments were independently repeated at least three times.

Scratch-wound healing assay. AGS and MGC-803 cells were transfected as indicated and allowed to grow to confluence. The cells were then cultured in the corresponding medium without serum for $12 \mathrm{~h}$ and scratched with a pipette tip. Wound areas were marked and photographed at 0 and $24 \mathrm{~h}$ 


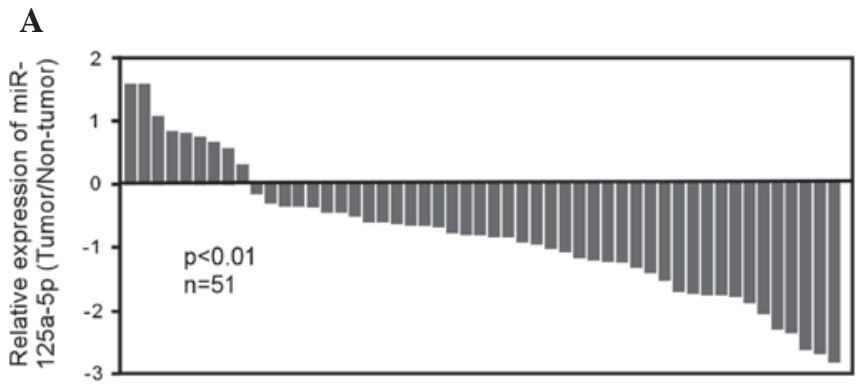

B

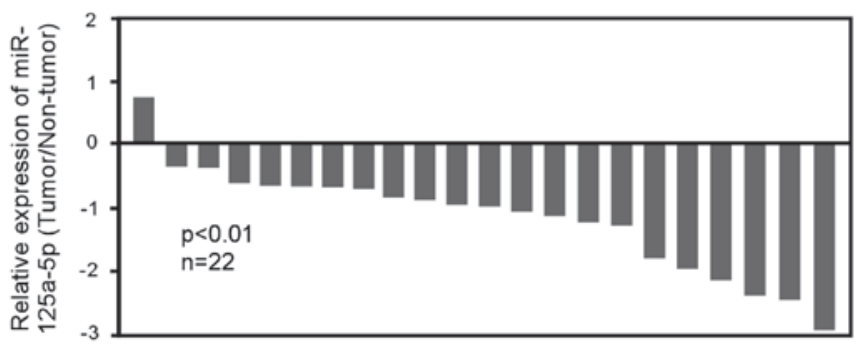

Figure 1. miR-125a-5p is significantly downregulated in human gastric cancer. (A) Relative expression of miR-125a-5p in 51 primary gastric cancer tissues compared with their pair-matched adjacent non-tumor tissues. (B) Relative expression of miR-125a-5p in 22 intestinal-type gastric cancer tissues compared with their matched non-tumor tissues. qRT-PCR was applied with specific primers for miR-125a-5p and snRNA U6. Data are shown as $-\Delta \Delta$ Ct values. P $<0.01$. qRT-PCR, quantitative real-time PCR.

using a phase-contrast microscope (Olympus IX81, Olympus Corporation), respectively. The rate of cell migration was evaluated by photographing and quantifying the migrated distance of cells moved from the wound edge toward the wound center using IPP 6.0 software (Media Cybernetics, Inc.). All experiments were repeated three times.

Matrigel invasion assay. For the Matrigel invasion assay, each 24-well insert with $8 \mu \mathrm{m}$ pore size was pre-coated with $50 \mu \mathrm{l}(1 \mu \mathrm{g} / \mu \mathrm{l})$ Matrigel (BD Biosciences, Bedford, MA, USA). AGS and MGC-803 cells were transfected with miR-125a-5p precursor or negative control as previously described. Following transfection (24 h), 50,000 cells were plated in $100 \mu \mathrm{l}$ serum-free medium in the upper Matrigelcoated chamber. The bottom chamber was placed with $600 \mu \mathrm{l}$ medium containing 20\% FBS. Following incubation for $12 \mathrm{~h}$ at $37^{\circ} \mathrm{C}$, the cells on the upper chamber were removed. Then, the cells in the bottom chamber were fixed, stained with DAPI for $2 \mathrm{~min}$, visualized under phase contrast microscope and photographed. The total number of invasive cells in nine randomly selected fields was counted by IPP software. All experiments were independently repeated at least three times.

Luciferase reporter assays. The E2F3 3'-UTR, containing the predicted miR-125a-5p binding site, was amplified and cloned into pMIR-REPORT vector (pMIR-E2F3) containing Firefly luciferase (Applied Biosystems). The binding site was also mutant and cloned into pMIR-REPORT vector (pMIR-E2F3-mut). Cells were cotransfected with pMIR-E2F3 or pMIR-E2F3-mut together with miR-125a-5p precursor or a negative control using siPORT amine transfection
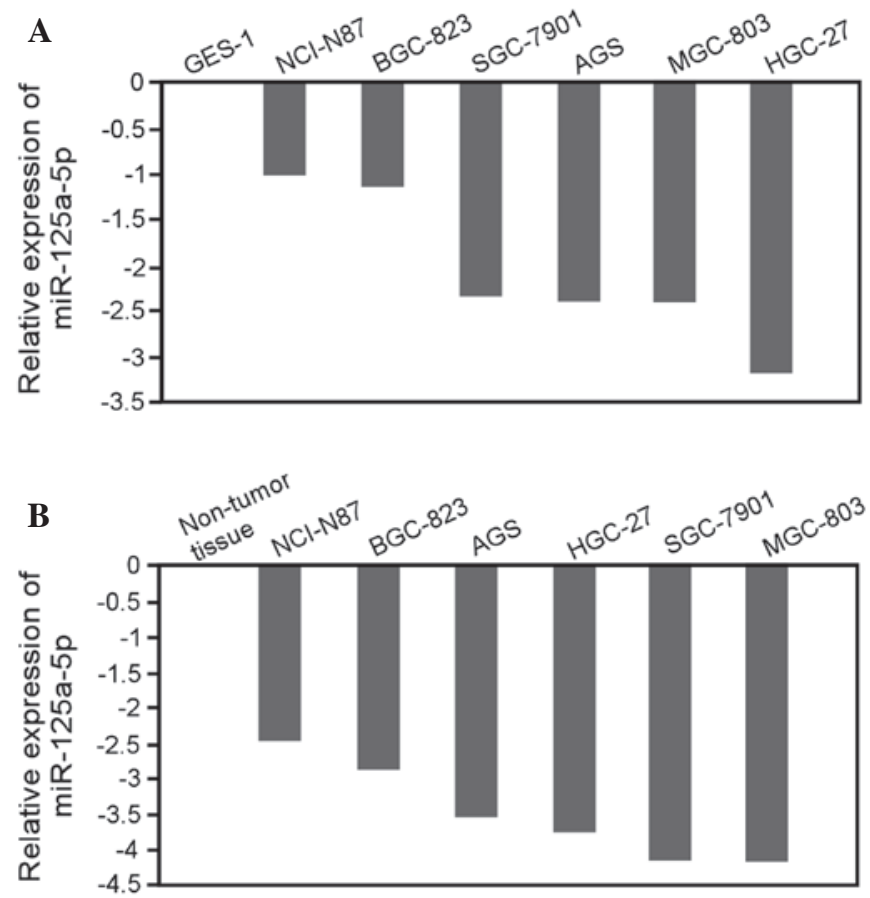

Figure 2. Expression of miR-125a-5p in gastric cancer cell lines. miR125a-5p expression was examined by TaqMan RT-PCR using snRNA U6 as a normalization control. The expression of miR-125a-5p in six different gastric cancer cell lines compared with either (A) non-malignant gastric epithelial cell line GES-1 or (B) a pool of three randomly chosen non-tumor gastric tissues from surgery. Data are presented as $\log 2$ value of miR-125a-5p in gastric cancer cell lines, normalized to GES-1 cells or non-tumor gastric tissues. RT-PCR, real-time PCR.

agent (Ambion). The pRL-TK vector containing Renilla luciferase was cotransfected as a reference control. Luciferase was measured by using dual-luciferase reporter assay (Promega Corporation, Madison, WI, USA).

Statistical analysis. Data are represented as the mean \pm standard error (SE) of at least three independent experiments. Student's t-test was performed to determine statistical significance. $\mathrm{P}<0.05$ was considered to indicate a statistically significant result.

\section{Results}

Downregulation of miR-125a-5p in primary gastric cancer tissues and cell lines. To investigate the expression profile of miRNAs in gastric cancer, an miRNA microarray was utilized to analyze five primary gastric cancer tissues compared with their matched non-tumor tissues. It was identified that miR125a-5p expression was markedly reduced in gastric cancer tissue. qRT-PCR analysis confirmed that miR-125a-5p was significantly downregulated in $\sim 82 \%$ of gastric tumor tissues (42 of 51 patients, $\mathrm{P}<0.01$ ), with 2.3 -fold reduction relative to their adjacent non-tumor tissues (Fig. 1A). Of note, the reduction of miR-125a-5p expression was demonstrated in 95.5\% of intestinal-type gastric cancer (21 of 22 patients, $\mathrm{P}<0.01)$ relative to their matched non-tumor tissues (Fig. 1B), whereas there was no significant change in miR-125a-5p expression in diffused-type and undetermined-type gastric cancer (data not shown). To further evaluate the correlation 
A

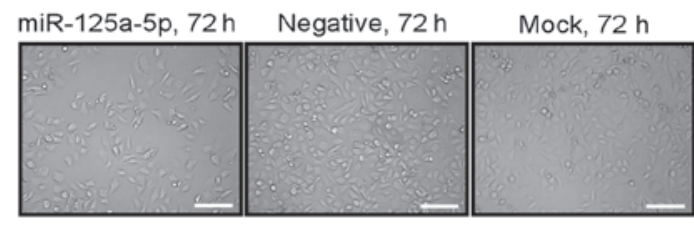

C

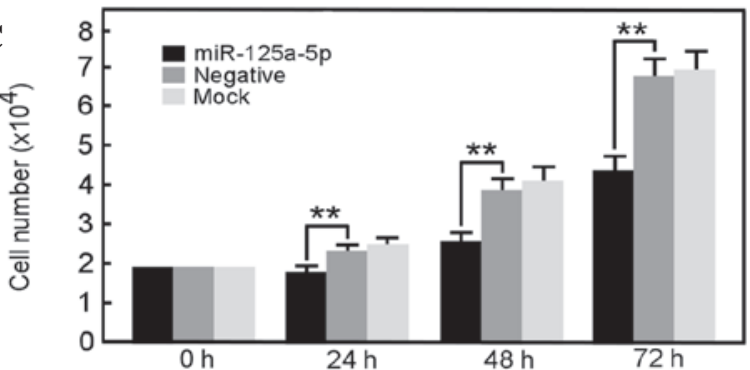

$\mathbf{E}$

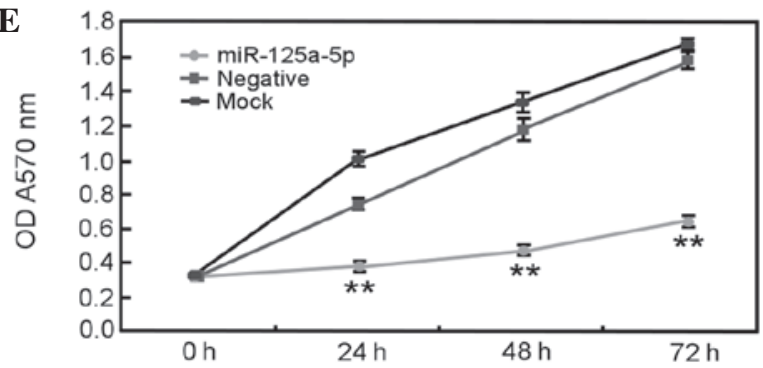

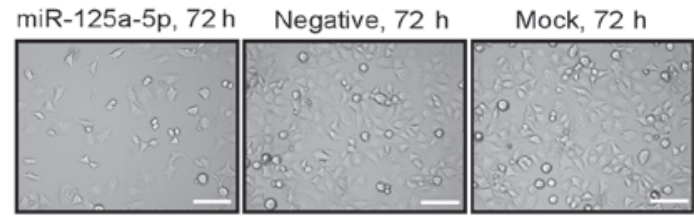

$\mathbf{D}$

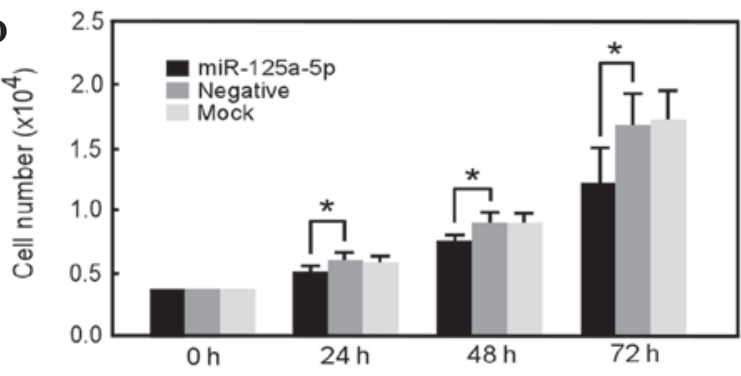

$\mathbf{F}$

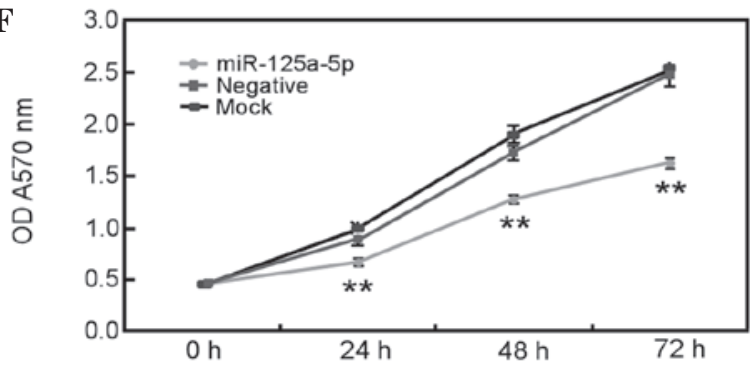

Figure 3. Effects of miR-125a-5p on the proliferation of AGS and MGC-803 cells. (A, C and E) AGS and (B, D and F) MGC-803 cells transfected with miR-125a-5p precursor, negative control or neither of the above were subjected to (A and B) phase-contrast microscopy, (C and D) cell count and (E and F) MTT analysis at the indicated time points, respectively. Scale bars, $50 \mu \mathrm{m}$. Data are presented as the mean $\pm \mathrm{SE}$ from three independent experiments. ${ }^{* *} \mathrm{P}<0.01$, compared with the negative control.. SE, standard error.

between miR-125a-5p expression and gastric cancer, we detected the expression of miR-125a-5p in six gastric cancer cell lines (AGS, BGC-823, HGC-27, MGC-803, NCI-N87 and SGC-7901 cells). The data revealed that the expression level of miR-125a-5p was significantly decreased in all cell lines derived from gastric cancers with various differentiation degrees, compared with the non-malignant gastric cell line GES-1 (Fig. 2A) or non-tumor stomach tissue (Fig. 2B). Taken together, these results indicate that miR-125a-5p is frequently downregulated in gastric cancer and therefore may be associated with gastric cancer progression.

Reduction of miR-125a-5p expression in gastric cancer is associated with distant metastasis. The correlation between the expression level of miR-125a-5p and the clinicopathological characteristics of gastric cancer are listed in Table I. A statistically significant association between miR-125a-5p expression level and distant metastasis was observed $(\mathrm{P}<0.05)$. However, no significant correlation was observed between miR-125a-5p expression level and gender, age, Lauren's classification, differentiation, node metastasis, invasion depth or stage. More clinical gastric cancer samples were required to further evaluate the correlation between miR-125a-5p expression and gastric cancer clinicopathological characteristics.

Overexpression of miR-125a-5p inhibits gastric cancer cell proliferation. To examine the potential role of miR-125a-5p in malignant phenotypes of gastric cancer cells, first, the effect of
miR-125a-5p overexpression on the proliferation of AGS and MGC-803 gastric cancer cells with low endogenous expression of miR-125a-5p was investigated. All of the results from phase contrast microscopy, direct cell count and MTT assays clearly demonstrated that overexpression of miR-125a-5p significantly inhibited the proliferation of AGS and MGC-803 cells (Fig. 3). Therefore, these results indicate that miR-125a-5p may have an important function in the proliferation of gastric cancer cells.

Overexpression of miR-125a-5p inhibits gastric cancer cell migration. The ability of cell migration and invasion are associated with cancer metastasis, so the role of miR-125a-5p in the migration and invasion of gastric cancer cells was investigated. The Transwell experiment demonstrated that miR-125a-5p overexpression greatly inhibited the migration of AGS (Fig. 4A and B) and MGC-803 cells (Fig. 4E and F). Furthermore, scratch wound healing assays revealed that the velocity of AGS (Fig. 4C and D) and MGC-803 (Fig. 4G and H) cell migration toward the wound area was also significantly reduced by overexpression of miR-125a-5p. Therefore, these data imply that miR-125a-5p may be important in gastric cancer cell migration.

Overexpression of miR-125a-5p inhibits gastric cancer cell invasion. Cell invasion into the surrounding tissue is a crucial, early step in gastric cancer development. Therefore, to determine whether miR-125a-5p affects the invasion ability of gastric cancer cells, we employed a Matrigel invasion assay. It was iden- 
A

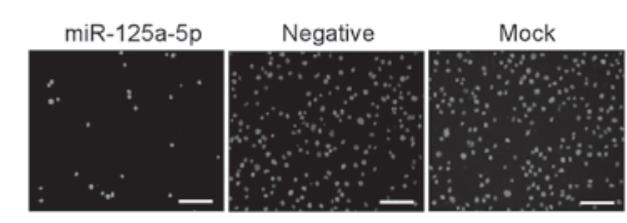

C

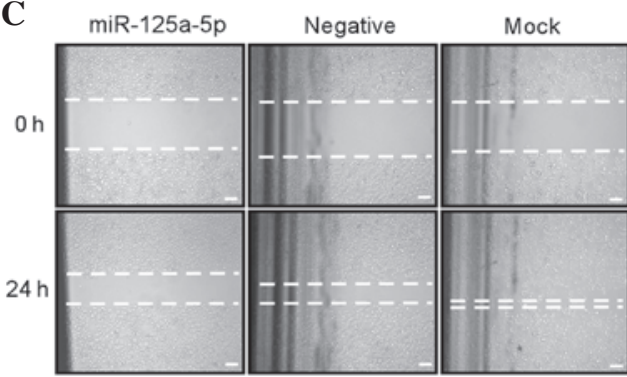

$\mathbf{E}$

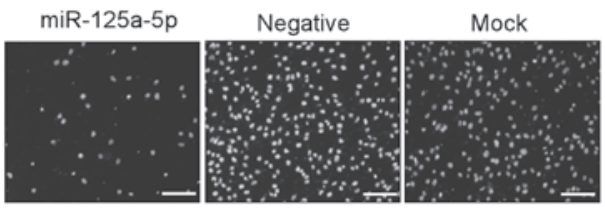

G

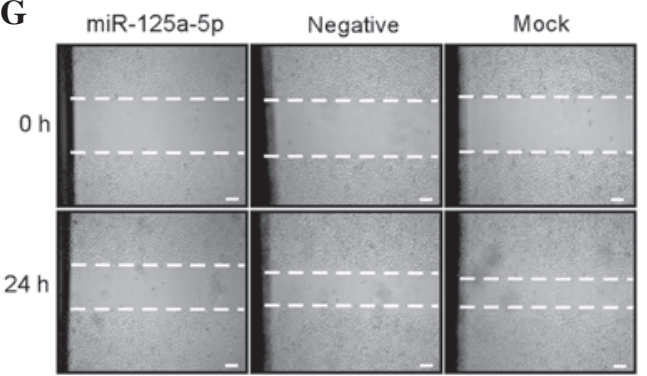

B

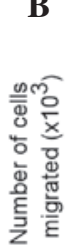

D
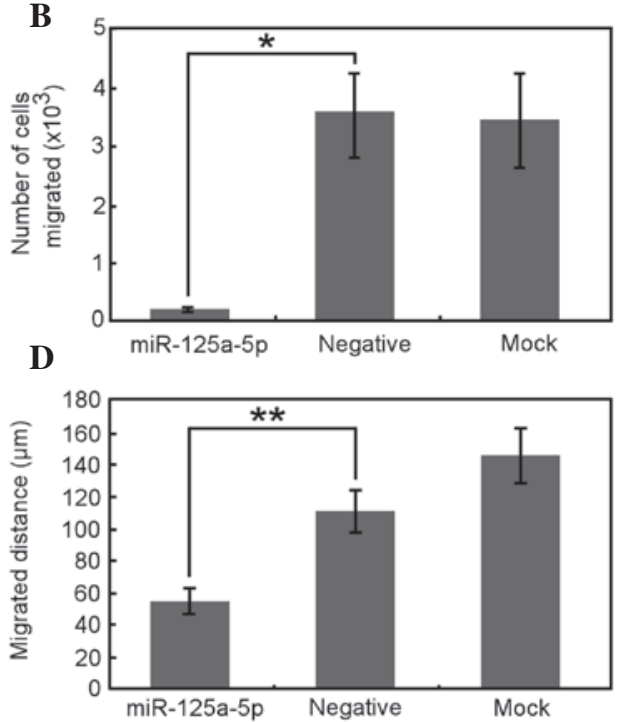

$\mathbf{F}$

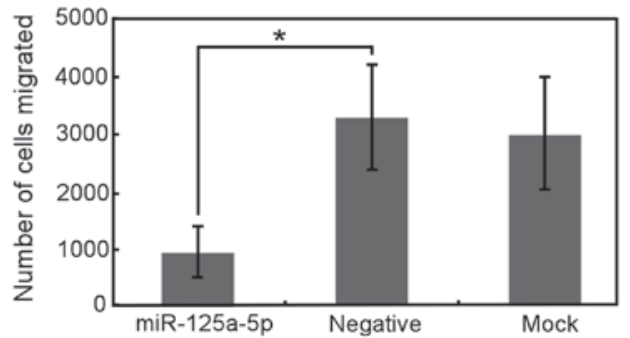

H

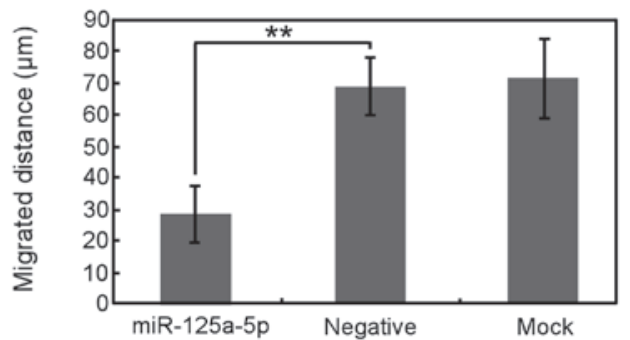

Figure 4. Overexpression of miR-125a-5p inhibits the migration of AGS and MGC-803 cells. (A-D) AGS and (E-H) MGC-803 cells transfected with miR-125a-5p precursor, negative control or neither of the above for 24 h were subjected to (A, B, E and F) Transwell and (C, D, G and H) scratch-wound healing analysis. (A and E) The cells that migrated to the bottom chamber were fixed and stained with DAPI. (B and F) Total number of migrated cells from nine randomly chosen fields was counted by IPP 6.0 software. (C and G) The cell migration to the wounded area was photographed by microscopy at 0 and $24 \mathrm{~h}$ post-wounding. The dotted lines indicate the areas lacking cells. (D and H) The rate of migration was examined by measuring the distance of cells moved from the wound edge toward the center following scratching. Data are presented as the mean $\pm \mathrm{SE}$ from at least three independent experiments. Bars, $50 \mu \mathrm{m}$. ${ }^{*} \mathrm{P}<0.05$ and ${ }^{* *} \mathrm{P}<0.01$. cp,[ared woth the negative control. DAPI, 4',6-diamidino-2-phenylindole; SE, standard error.

tified that miR-125a-5p overexpression significantly inhibited the invasion ability of AGS and MGC-803 cells (Fig. 5). These results indicate that downregulation of $\mathrm{miR}-125 \mathrm{a}-5 \mathrm{p}$ in gastric cancer may be sufficient to promote gastric cancer invasion. In the two cell lines, ectopic expression of miR-125a-5p did result in a 2- to 4-fold decrease in cell migration or invasiveness.

miR $-125 a-5 p$ regulates E2F3 through 3 '-UTR. To assess the mechanism of miR-125a-5p function in gastric cancer cell proliferation, migration and invasion, by using prediction tools including miRanda (mirSVR; http://www.microrna .org/ microrna/home.do), TargetScan and Pictar algorithms, the potential targets of miR-125a-5p were screened. Among the hundreds of targets that were predicted, E2F3 was further studied as a potential target. E2F3 is regarded as an oncogene because it is amplified in various human tumor types. To obtain direct evidence that E2F3 is a potential target of miR-125a-5p, we examined whether the predicted binding sites of miR-125a-5p in the 3'-UTR of E2F3 mRNA were responsible for its regulation. The 3'-UTR of E2F3 downstream of a luciferase reporter gene (pMIR-E2F3) was cloned, and this vector was co-transfected with an miR-125a-5p precursor or its negative control into gastric cancer cells. The luciferase activity of cells transfected with miR-125a-5p precursor was significantly reduced compared with the negative control $(\mathrm{P}<0.01$; Fig. 6$)$. Furthermore, mutation of the putative binding site clearly abrogated the repression of luciferase activity caused by miR-125a-5p overexpression. These data suggest that miR-125a-5p may inhibit E2F3 expres- 
A

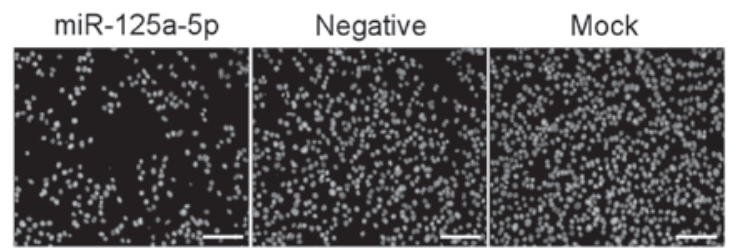

B

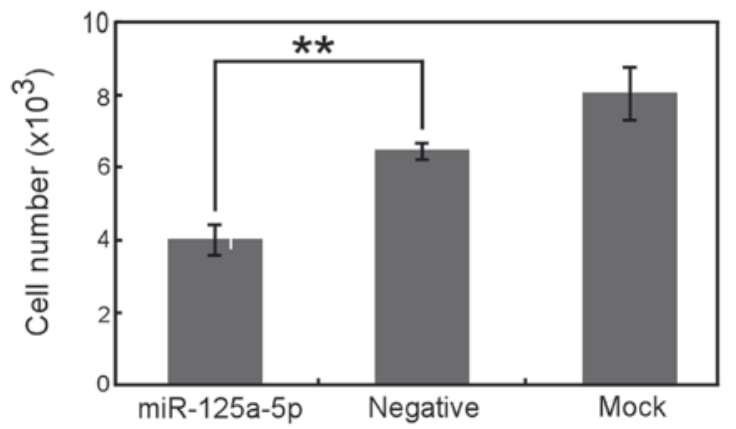

C

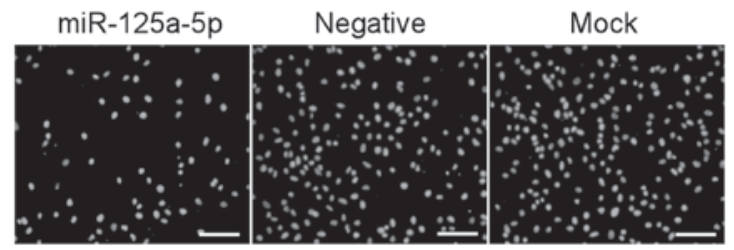

D

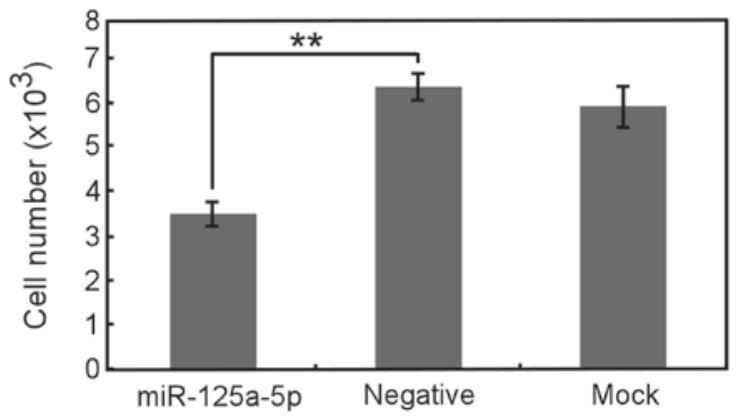

Figure 5. Overexpression of miR-125a-5p inhibits the invasion of AGS and MGC-803 cells. (A and B) AGS and (C and D) MGC-803 cells transfected with $\mathrm{miR}-125 \mathrm{a}-5 \mathrm{p}$ precursor, negative control or neither of the above were subject to Matrigel invasion analysis. (A and C) Representative fields of the cells on the bottom chamber at $12 \mathrm{~h}$ post-invasion were demonstrated. Scale bars, $50 \mu \mathrm{m}$. (B and D) The total number of invasive cells from nine randomly selected fields were counted by IPP 6.0. Data are represented as the mean \pm SE from three independent experiments. ${ }^{* *} \mathrm{P}<0.01$, compared with the negative control. SE, standard error.

sion through 3'-UTR at the post-transcriptional level. Further investigations are required to examine the association between the expression of miR-125a-5p and E2F3 in gastric cancer tissues.

\section{Discussion}

Recent studies have established the presence of miRNA expression signatures in gastric cancer, but our understanding of the function of aberrant miRNAs in gastric carcinoma progression remains in its infancy. Our presented data demonstrate a potential role for miR-125a-5p in gastric cancer. In our previous study, we aimed to examine the miRNA expression profile in gastric cancer by using miRNA microarray techniques, and it was identified that miR-125a-5p is one of the most downregulated

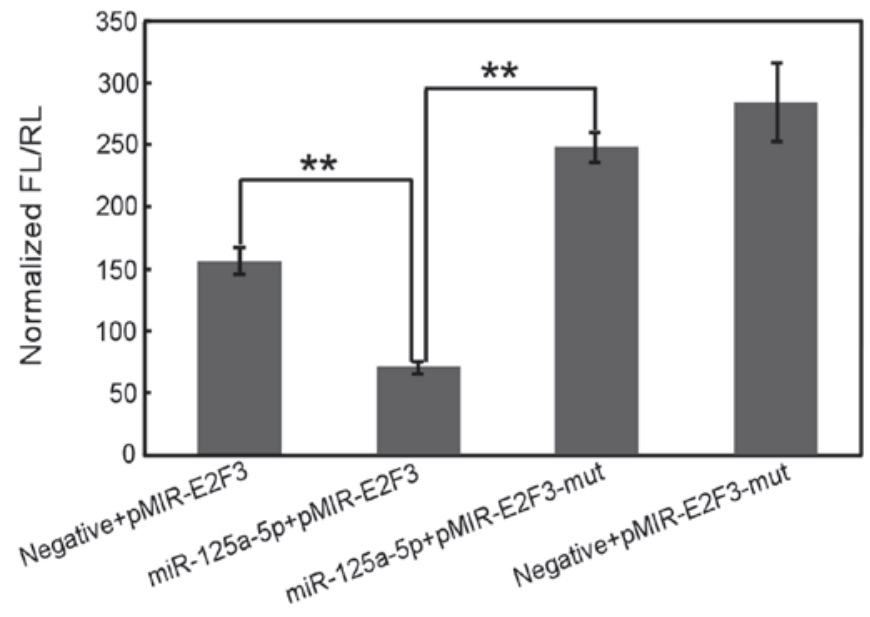

Figure 6. miR-125a-5p targeted E2F3 by binding to its 3'-UTR. Gastric cancer cells were cotransfected with miR-125a-5p precursor and a luciferase reporter containing a fragment of the 3'-UTR of E2F3 harboring either the miR-125a-5p binding site (pMIR-E2F3) or a mutant site (pMIR-E2F3-mut). The assay revealed that the luciferase activity in E2F3 group was significantly decreased compared with the luciferase activities in the mutant and negative control groups. ${ }^{*} \mathrm{P}<0.05$. 3'-UTR region, 3 '-untranslated region.

miRNAs in gastric cancer. In the present study, it was revealed that miR-125a-5p was significantly downregulated in $>80 \%$ of gastric cancer samples compared with their adjacent non-tumor tissues obtained from patients undergoing gastric resection, which was further confirmed by the data from gastric cancer cell lines. Restoration of miR-125a-5p expression substantially inhibited proliferation, migration and invasion activities of gastric cancer cells. These results suggest a potential role of miR-125a-5p in gastric carcinogenesis.

miR-125a-5p has been identified to be downregulated in a number of malignancies, including lung, head and neck, gastric and esophageal and hepatocellular carcinogenesis (27-31), which are consistent with the results of our study. In vitro assays revealed that HER2 is a direct target of miR-125a-5p, which potently suppresses the proliferation of gastric cancer cells $(28,31)$. Whereas, limited data is available identifying the targets of miR-125a-5p involved in gastric cancer cells migration and invasion.

As part of our investigations into how the downregulation of miR-125a-5p expression affects gastric cancer progression, a number of the prediction algorithms were employed, including PicTar, TargetScan and miRanda (32-34), to search putative targets of miR-125a-5p. Since the overexpression of miR-125a-5p inhibits cell proliferation, migration and invasion, it is possible to expect that the target genes of miR-125a-5p may have oncogenic characteristics. Among the hundreds of targets that were predicted, E2F3 is regarded as an oncogene because it is amplified in various human tumor types, including lung, bladder, prostate, colon and breast cancer, and is involved in cancer cells apoptosis and proliferation (35-39). Furthermore, E2F3 amplification and overexpression is demonstrated to be associated with cell migration and invasion with comprehensive mechanisms (40-42). For example, the data of Oeggerli's et al study concluded that E2F3 was frequently amplified and overexpressed in invasively growing bladder cancer, which indicated an important role of E2F3 in cancer progression (42). In the present study, using the Luciferase assay, direct evidence was 
obtained that E2F3 is potentially a direct target of miR-125a-5p. These data demonstrated that miR-125a-5p significantly inhibited the luciferase activity of pMIR-E2F3 which contained the 3'-UTR of E2F3 but had no effect on pMIR-E2F3-mut, which accommodated the mutant 3'-UTR of E2F3. Together, it was hypothesized that the downregulation of miR-125a-5p results in overexpression of E2F3 which subsequently contributes to gastric cancer progression.

Additionally, these data reveal that miR-125a-5p is significantly downregulated in the majority of the intestinal-type gastric cancer samples ( 21 of 22 patients; $\mathrm{P}<0.05$ ), implying a highly possible association between the expression level of miR-125a-5p and the Lauren's classification of gastric cancer. A statistically significant association between miR-125a-5p expression level and distant metastasis was also observed $(\mathrm{P}<0.05)$. However, no significant correlation was observed between the expression level of miR-125a-5p and gender, age, Lauren's classification, differentiation, node metastasis, invasion depth or stage. To confirm the association between miR-125a-5p expression and the clinicopathological characteristics of gastric cancer, future studies should employ a larger sample, to further analyze the association of miR-125a-5p expression and the clinical outcomes of gastric cancer.

The identification of the expression levels and tumorsuppressive function of miR-125a-5p in gastric cancer types, provides a new window of therapeutic opportunity. The development of modified miRNAs with longer half-time and higher efficiency has produced favorable anticancer outcomes in experimental models, including the locked nucleic acidmodified oligonucleotides and the antisense oligonucleotides termed 'antagomirs' (43-45). Therefore, enforced expression of miR-125a-5p utilizing approaches such as transfection of miR-125a-carrying viruses or synthetic miR-125a-5p oligonucleotides, will be required for future study in gastric carcinoma pathology.

\section{Acknowledgements}

This study was supported by Natural Scientific Foundation of China (nos. 81402429, 30771107 and 30901714), and the Natural Scientific Foundation of Zhejiang Province, China (nos. LQ14H160003, Z2100247 and Y2100106).

\section{References}

1. Bartel DP: MicroRNAs: genomics, biogenesis, mechanism, and function. Cell 116: 281-297, 2004.

2. Calin GA, Sevignani C, Dumitru CD, et al: Human microRNA genes are frequently located at fragile sites and genomic regions involved in cancers. Proc Natl Acad Sci USA 101: 2999-3004, 2004.

3. Li X, Zhang Y, Zhang Y, Ding J, Wu K and Fan D: Survival prediction of gastric cancer by a seven-microRNA signature. Gut 59: 579-585, 2010.

4. Kong KL, Kwong DL, Chan TH, et al: MicroRNA-375 inhibits tumour growth and metastasis in oesophageal squamous cell carcinoma through repressing insulin-like growth factor 1 receptor. Gut 61: 33-42, 2012.

5. Hsu KW, Wang AM, Ping YH, et al: Down-regulation of tumor suppressor MBP-1 by microRNA-363 in gastric carcinogenesis. Carcinogenesis: Oct 4, 2013 (Epub ahead of print).

6. Peng Y, Dai Y, Hitchcock C, et al: Insulin growth factor signaling is regulated by microRNA-486, an underexpressed microRNA in lung cancer. Proc Natl Acad Sci USA 110: $15043-$ 15048,2013
7. Zhang N, Wang X, Huo Q, et al: MicroRNA-30a suppresses breast tumor growth and metastasis by targeting metadherin. Oncogene: Jul 15, 2013 (Epub ahead of print).

8. Volinia S, Galasso M, Sana ME, et al: Breast cancer signatures for invasiveness and prognosis defined by deep sequencing of microRNA. Proc Natl Acad Sci USA 109: 3024-3029, 2012.

9. Li D, Liu X, Lin L, et al: MicroRNA-99a inhibits hepatocellular carcinoma growth and correlates with prognosis of patients with hepatocellular carcinoma. J Biol Chem 286: 36677-36685, 2011.

10. Osada $\mathrm{H}$ and Takahashi T: let-7 and miR-17-92: small-sized major players in lung cancer development. Cancer Sci 102: 9-17, 2011.

11. Aqeilan RI, Calin GA and Croce CM: miR-15a and miR-16-1 in cancer: discovery, function and future perspectives. Cell Death Differ 17: 215-220, 2010.

12. Wiklund ED, Bramsen JB, Hulf T, et al: Coordinated epigenetic repression of the miR-200 family and miR-205 in invasive bladder cancer. Int J Cancer 128: 1327-1334, 2011.

13. Parkin DM, Bray F, Ferlay J and Pisani P: Global cancer statistics, 2002. CA Cancer J Clin 55: 74-108, 2005.

14. Hohenberger P and Gretschel S: Gastric cancer. Lancet 362: 305-315, 2003.

15. Wu Q, Jin H, Yang Z, et al: MiR-150 promotes gastric cancer proliferation by negatively regulating the pro-apoptotic gene EGR2. Biochem Biophys Res Commun 392: 340-345, 2010.

16. Liu T, Tang H, Lang Y, Liu M and Li X: MicroRNA-27a functions as an oncogene in gastric adenocarcinoma by targeting prohibitin. Cancer Lett 273: 233-242, 2009.

17. Chan $\mathrm{SH}, \mathrm{Wu} \mathrm{CW}, \mathrm{Li} \mathrm{AF}, \mathrm{Chi} \mathrm{CW}$ and Lin WC: miR-21 microRNA expression in human gastric carcinomas and its clinical association. Anticancer Res 28: 907-911, 2008.

18. Kim YK, Yu J, Han TS, et al: Functional links between clustered microRNAs: suppression of cell-cycle inhibitors by microRNA clusters in gastric cancer. Nucleic Acids Res 37: 1672-1681, 2009.

19. Gao C, Zhang Z, Liu W, Xiao S, Gu W and Lu H: Reduced microRNA-218 expression is associated with high nuclear factor kappa B activation in gastric cancer. Cancer 116: 41-49, 2010.

20. Luo H, Zhang H, Zhang Z, et al: Down-regulated miR-9 and miR-433 in human gastric carcinoma. J Exp Clin Cancer Res 28: $82,2009$.

21. Takagi T, Iio A, Nakagawa Y, Naoe T, Tanigawa N and Akao Y: Decreased expression of microRNA-143 and -145 in human gastric cancers. Oncology 77: 12-21, 2009.

22. Ding L, Xu Y, Zhang W, et al: MiR-375 frequently downregulated in gastric cancer inhibits cell proliferation by targeting JAK2. Cell Res 20: 784-793, 2010.

23. Pan J, Hu H, Zhou Z, et al: Tumor-suppressive mir-663 gene induces mitotic catastrophe growth arrest in human gastric cancer cells. Oncol Rep 24: 105-112, 2010.

24. Zhang Z, Li Z, Gao C, et al: miR-21 plays a pivotal role in gastric cancer pathogenesis and progression. Lab Invest 88: 1358-1366, 2008.

25. Tsukamoto Y, Nakada C, Noguchi T, et al: MicroRNA-375 is downregulated in gastric carcinomas and regulates cell survival by targeting PDK1 and 14-3-3zeta. Cancer Res 70: 2339-2349, 2010.

26. Du Y, Xu Y, Ding L, et al: Down-regulation of miR-141 in gastric cancer and its involvement in cell growth. J Gastroenterol 44: 556-561, 2009.

27. Jiang L, Huang Q, Chang J, Wang E and Qiu X: MicroRNA HSA-miR-125a-5p induces apoptosis by activating p53 in lung cancer cells. Exp Lung Res 37: 387-398, 2011.

28. Nishida N, Mimori K, Fabbri M, et al: MicroRNA-125a-5p is an independent prognostic factor in gastric cancer and inhibits the proliferation of human gastric cancer cells in combination with trastuzumab. Clin Cancer Res 17: 2725-2733, 2011.

29. Odar K, Boštjančič E, Gale N, Glavač D and Zidar N: Differential expression of microRNAs miR-21, miR-31, miR-203, miR-125a-5p and miR-125b and proteins PTEN and p63 in verrucous carcinoma of the head and neck. Histopathology 61: 257-265, 2012.

30. Kim JK, Noh JH, Jung KH, et al: Sirtuin7 oncogenic potential in human hepatocellular carcinoma and its regulation by the tumor suppressors MiR-125a-5p and MiR-125b. Hepatology 57: 1055-1067, 2013.

31. FassanM,PizziM,RealdonS,etal:TheHER2-miR125a5p/miR125b loop in gastric and esophageal carcinogenesis. Hum Pathol 44: 1804-1810, 2013

32. Krek A, Grun D, Poy MN, et al: Combinatorial microRNA target predictions. Nat Genet 37: 495-500, 2005.

33. Lewis BP, Shih IH, Jones-Rhoades MW, Bartel DP and Burge CB: Prediction of mammalian microRNA targets. Cell 115: 787-798, 2003. 
34. John B, Enright AJ, Aravin A, Tuschl T, Sander C and Marks DS Human MicroRNA targets. PLoS Biol 2: e363, 2004.

35. Cooper CS, Nicholson AG, Foster C, et al: Nuclear overexpression of the E2F3 transcription factor in human lung cancer. Lung Cancer 54: 155-162, 2006.

36. Feber A, Clark J, Goodwin G, et al: Amplification and overexpression of E2F3 in human bladder cancer. Oncogene 23 1627-1630, 2004.

37. Foster CS, Falconer A, Dodson AR, et al: Transcription factor $\mathrm{E} 2 \mathrm{~F} 3$ overexpressed in prostate cancer independently predicts clinical outcome. Oncogene 23: 5871-5879, 2004.

38. Fang Y, Gu X, Li Z, Xiang J and Chen Z: miR-449b inhibits the proliferation of SW1116 colon cancer stem cells through downregulation of CCND1 and E2F3 expression. Oncol Rep 30: 399-406, 2013.

39. Vimala K, Sundarraj S, Sujitha MV and Kannan S: Curtailing overexpression of E2F3 in breast cancer using siRNA (E2F3)based gene silencing. Arch Med Res 43: 415-422, 2012.
40. McClellan KA, Ruzhynsky VA, Douda DN, et al: Unique requirement for $\mathrm{Rb} / \mathrm{E} 2 \mathrm{~F} 3$ in neuronal migration: evidence for cell cycle-independent functions. Mol Cell Biol 27: 4825-4843, 2007.

41. Ziebold U, Reza T, Caron A and Lees JA: E2F3 contributes both to the inappropriate proliferation and to the apoptosis arising in $\mathrm{Rb}$ mutant embryos. Genes Dev 15: 386-391, 2001.

42. Oeggerli M, Tomovska S, Schraml P, et al: E2F3 amplification and overexpression is associated with invasive tumor growth and rapid tumor cell proliferation in urinary bladder cancer. Oncogene 23: 5616-5623, 2004

43. Ørom UA, Kauppinen S and Lund AH: LNA-modified oligonucleotides mediate specific inhibition of microRNA function. Gene 372: 137-141, 2006.

44. Surdziel E, Eder M and Scherr M: Lentivirus-mediated antagomir expression. Methods Mol Biol 667: 237-248, 2010.

45. Velu CS and Grimes HL: Utilizing antagomiR (antisense microRNA) to knock down microRNA in murine bone marrow cells. Methods Mol Biol 928: 185-195, 2012. 